\title{
Synthesis and thermal degradation characterization of novel poly(phosphazene-aryl amides)
}

\author{
Z. P. Zhao, Q. Guo ${ }^{*}$, X. Li, J. L. Sun, Z. J. Nie \\ School of Materials Science and Engineering, Shanghai University, Shanghai 201800, China
}

Received 18 August 2011; accepted in revised form 1 November 2011

\begin{abstract}
New fully aromatic poly(phosphazene-aryl amides) were prepared by polycondensation reaction of our synthesized aromatic diamine: 1,1,3,5-tetraphenoxy-4,6-bis(4-aminophenoxy)oligocyclotriphosphazene (monomer 1) with terephthaloyl dichloride. Their chemical structure and composition were characterized by elemental analysis, ${ }^{1} \mathrm{H}$ and ${ }^{31}$ P NMR (Nuclear Magnetic Resonance), and FT-IR (Fourier transform infrared) spectroscopy, whereas their thermal degradation properties were determined by DSC (Differential Scanning Calorimetry) and TGA (Thermal Gravimertic Analysis) techniques. The solid residues of all samples were analysed by FT-IR and SEM (Scanning Electron Microscopy). Compared to conventional PPTA (poly(p-phenylene terephthamide)), PPAA (poly(phosphazene-aryl amide)) shows excellent thermal stability and solubility in polar protic solvents. All poly(phosphazene-aryl amides) show two thermal degradation in the temperature range $150-600^{\circ} \mathrm{C}$. The monomer 1 , due to its structure, shows the first maximum rate of thermal decomposition temperature around $150-350^{\circ} \mathrm{C}$, which may be due to the decomposition of the $\mathrm{P}-\mathrm{O}-\mathrm{C}$ bone. Morphology of the solid residues by Scanning Electron Microscope exhibit that the granular of the solid residues gradual disappearance with the increase of monomer 1 content. The surface layer of PPAA solid residues has been grumous, for the syneresis of $\mathrm{P}-\mathrm{O}-\mathrm{P}$ took place.
\end{abstract}

Keywords: polymer composites, phosphazene, polyamide, thermal degradation

\section{Introduction}

Polyamides are considered to be high-performance organic materials due to their outstanding thermal and mechanical properties [1-3]. Their properties arise from their aromatic structure and amide linkages. However, the poor solubility, high softening and melting temperatures caused by high crystallinity and high stiffness of polymer backbone hamper the processing of aromatic polyamides. What's more, the properties can be easily tailored through changing their molecular chain structure [4-6]. The strategies which have been employed for solubility increase in aromatic polyamides are lateral substitution and the distortion of the rigid character introducing non-planar, kinked or flexible parts in the main chain. Research efforts are there- fore underway to take advantage of their properties, enhance their machinability, solubility and incorporate new chemical functionalities in the polyamide backbone or lateral structure, so that their applicability is expanded and remains on the forefront of scientific research [7-9].

Vapor phase lubrication has been proposed as a scheme for lubrication of high efficiency engines operating at temperature outside the range in which any fluid lubricants can be used [10-16]. These applications include gas-turbines which operate with bearing temperatures in excess of $500^{\circ} \mathrm{C}$. The most commonly used vapor phase lubricants for these applications are the aryl phosphates and in particular tricresyl phosphate [17].

\footnotetext{
${ }^{*}$ Corresponding author, e-mail: guoq@shu.edu.cn
}

(c) BME-PT 
In our research, we decide to synthesize a new aromatic phosphazene diamine monomer 1 and use it for the synthesis of new fully aromatic poly(phosphazene-aryl amides), in order to investigate their thermal behavior. These data will be fundamental for the following syntheses of known and commercial polyamides containing different $\mathrm{mol} \%$ of monomer 1. Also its will be used as the additiontype monomers selected on the basis of the tribopolymerization concept in Vapor phase lubrication study. Thus, in the present work we report the synthesis and characterization either of the monomer 1 and their fully aromatic polyamides. Their physical chemistry properties including intrinsic viscosity, solubility behavior, crystallization behavior, thermal properties and degradation behavior are studied.

\section{Experimental}

\subsection{Materials}

Hexachlorocyclotriphosphazene (HCCP, self-made) was purified by recrystallization from dry n-heptane and subsequent sublimation twice [18]. 4, 4-diaminodiphenyl ether (ODA) was used as received from Sinopharm Chemical Regents Co. Ltd (99\%, Shanghai, China). Phenol was recrystallized from pentane. Tetrahydrofurane (THF) was dried over and distilled from sodium/benzophenone. Triethylamine (TEA) was distilled onto molecular sieves. N-methyl2-pyrrolidinone (NMP) was dried over phosphorous pentoxide and distilled under reduced pressure. Terephthaloyl chloride (TPC) was purified by recrystallization form anhydrous hexane. pPhenylenediamine (PPD) was purified by vacuum distillation. Lithium chloride ( $\mathrm{LiCl})$ was dried for $8 \mathrm{~h}$ at 170 under vacuum. All chemicals and solvents were provided commercially by Sinopharm Chemical Regents Co. Ltd (China) and used without further purification unless otherwise noted. All glassware was dried in an oven under vacuum before use.

\subsection{Synthesis of 1,1,3,5-tetraphenoxy-4,6-bis(4- aminophenoxy)oligocyclotriphosphazene (monomer 1)}

The preparation of monomer 1 involved two steps: In the first step, $\mathrm{NaH}(9.6 \mathrm{~g}, 0.40 \mathrm{~mol})$ was dissolved in $20 \mathrm{ml}$ THF under stirring and nitrogen atmosphere. To this, a solution of THF (50 ml) dis- solving phenol (37.65 g, $0.40 \mathrm{~mol})$ was added dropwise. The temperature of reaction mixture was maintained at room temperature for 2 hours and then added this solution dropwise to a solution of HCCP $(34.8 \mathrm{~g}, 0.10 \mathrm{~mol})$ in $100 \mathrm{ml}$ of THF at room temperature for 24 hours. Then, after THF removed by rotary evaporation, the reaction mixture was to be purified by ethanol and deionized water three times. Finally, the product I (bis-chlorinetetraphenoxycyclotriphosphazene) was dried under vacuum at $60 \mathrm{C}$ for $12 \mathrm{~h}$. In the second step, a solution of the product I $(45.25 \mathrm{~g}, 0.050 \mathrm{~mol})$ in $100 \mathrm{ml}$ of THF was added dropwise to a solution of ODA ( $20 \mathrm{~g}$, $0.10 \mathrm{~mol})$ and TEA $(10.05 \mathrm{~g}, 0.10 \mathrm{~mol})$ in $100 \mathrm{ml}$ of THF at room temperature. After completion of the addition in $2 \mathrm{~h}$, the mixture was stirred and refluxed at $40^{\circ} \mathrm{C}$ for $12 \mathrm{~h}$. Then, the reaction mixture was cooled to room temperature. THF was removed by distillation under reduced pressure. The residue was washed with methanol and deionized water three times, and then dried over anhydrous magnesium sulfate. Finally, the product II (monomer 1) was dried under vacuum at $60^{\circ} \mathrm{C}$.

FTIR $\left[\mathrm{cm}^{-1}\right.$ ]: $3325\left(v_{\mathrm{N}-\mathrm{H}}\right), 1580\left(v_{\mathrm{Ph}}\right), 1160\left(v_{\mathrm{C}-\mathrm{O}-\mathrm{C}}\right)$, $1230\left(v_{\mathrm{P}=\mathrm{N}}\right), 940\left(v_{\mathrm{P}-\mathrm{O}-\mathrm{Ph}}\right), 875\left(v_{\mathrm{P}-\mathrm{N}}\right) .{ }^{1} \mathrm{H}$ NMR (DMSO; $\delta, \mathrm{ppm}): 7.65-7.96\left(\mathrm{~d}, \mathrm{C}_{6} \mathrm{H}_{4}\right.$ in ODA), 6.88-7.21 (t, $\mathrm{C}_{6} \mathrm{H}_{5}$ in phenoxy), $5.91\left(\mathrm{~s}, \mathrm{NH}_{2}\right), 3.04$ (s, NH). ${ }^{31} \mathrm{P}$ NMR (DMSO; $\left.\delta, \quad \mathrm{ppm}\right): 7.96$ $(\mathrm{PPh}(\mathrm{ODA})), 5.64\left(\mathrm{PPh}_{2}\right)$.

\subsection{Synthesis of poly(phosphazene-aryl amides)}

In a typical experiment, polyamide PPAA, which derived from monomer $\mathbf{1}$ and TPC, was prepared as follows: A two-necked flask equipped with a dropping funnel and gas inlet tube was charged with a solution of monomer $1(9.21 \mathrm{~g}, 0.01 \mathrm{~mol})$ and pyridine $(1.55 \mathrm{~g})$ in $20 \mathrm{ml}$ of NMP, to which TPC $(2.04 \mathrm{~g}$, $0.01 \mathrm{~mol}$ ) was added with stirring under $\mathrm{N}_{2}$. The mixture was firstly stirred at $0^{\circ} \mathrm{C}$ for $0.5 \mathrm{~h}$, then at room temperature for $7 \mathrm{~h}$ to yield a viscous polyamide solution. Then the solution was trickled into excess ethanol with stirring to afford a precipitate. The precipitate was filtered, washed with hot ethanol for three times, purified by Soxhlet extractor (Acetone as solvent) for $12 \mathrm{~h}$, then washed three times with deionized water and dried at $120^{\circ} \mathrm{C}$ under vacuum for $6 \mathrm{~h}$ to give polyamide PPAA (yield: 88.2\%). Other polyamides, PPTA (yield: $93.4 \%$ ), 


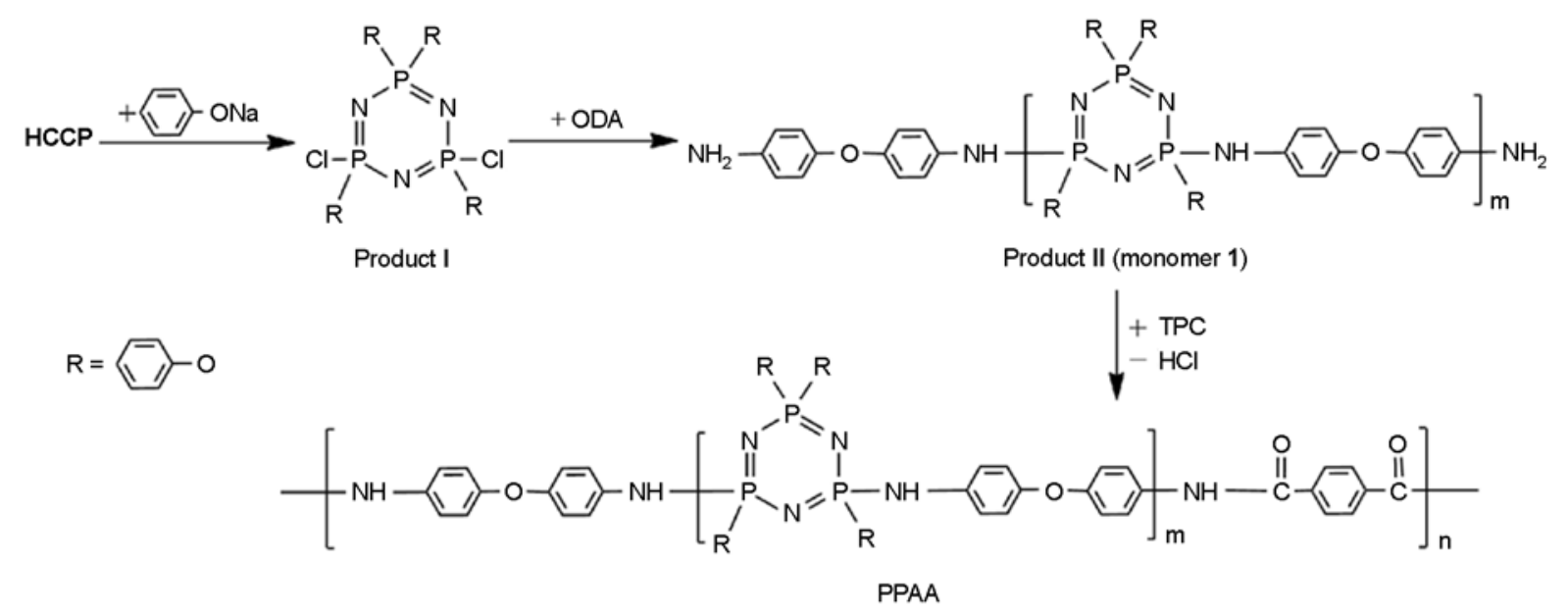

Figure 1. Synthesis of monomer 1 and poly(phosphazene-aryl amides)

Table 1. The chemical compositions of polyamides

\begin{tabular}{|l|c|c|c|}
\hline $\begin{array}{c}\text { Polymer } \\
\text { code }\end{array}$ & $\begin{array}{c}\text { PPD } \\
\text { [molar percent] }\end{array}$ & $\begin{array}{c}\text { Monomer 1 } \\
\text { [molar percent] }\end{array}$ & $\begin{array}{c}\text { TPC } \\
\text { [molar percent] }\end{array}$ \\
\hline PPTA & 100 & 0 & 100 \\
\hline PA-7030 & 70 & 30 & 100 \\
\hline PA-5050 & 50 & 50 & 100 \\
\hline PA-3070 & 30 & 70 & 100 \\
\hline PPAA & 0 & 100 & 100 \\
\hline
\end{tabular}

PA-7030 (yield: $89.2 \%$ ), PA-5050 (yield: 91.6\%), PA-3070 (yield: $88.7 \%$ ), were synthesized by similar procedure. The chemical compositions of the polyamides are listed in Table 1. All composites described were synthesized by similar methods as Figure 1.

\subsection{Measurements}

FT-IR spectra of all samples were recorded using polymer granule on a Perkin-Elmer Wellesley MA spectrophotometer. The $\left({ }^{1} \mathrm{H}\right.$ and $\left.{ }^{31} \mathrm{P}\right)$ NMR spectra were recorded on a Varian DRX 400 NMR spectrometer (Varian NMR Instruments) with the operating frequency at $400 \mathrm{MHz}$ using $\mathrm{CDCl}_{3}$ or DMSO as a solvent, using TMS as inner reference and $\mathrm{H}_{3} \mathrm{PO}_{4}(85 \%)$ as external reference. Elemental analysis was carried out using a Heraeus $\mathrm{CHN}-\mathrm{O}$ rapid elemental analyzer (United States) with acetanilide as a standard. The intrinsic viscosities of polyamides were measured at a concentration of $0.5 \mathrm{~g} / \mathrm{dl}$ in concentrated sulfuric acid, at $30 \pm 0.2$. The numbers were counted by measured value from suspended level Ubbelohde viscometer. Gel permeation chromatographic analysis (GPC) was carried out on a PL GPC 50 plus evaporative mass detector instrument
(United States). Dimethylformamide (DMF) was used as mobile phase after calibration with polystyrene standards of known molecular weights. Thermogravimetric analysis (TGA) was performed on a TGA 7 instrument (PerkinElmer) thermal analysis system. Sample weight taken was $2-4 \mathrm{mg}$. DSC analysis was carried out on a Perkin-Elmer Pyris 2 DSC analyzer (PerkinElmer), at a heating rate of $10^{\circ} \mathrm{C} / \mathrm{min}$ in nitrogen atmosphere. Sample weight taken was $15-20 \mathrm{mg}$. Wide-angle X-ray scattering measurements were performed on a Bruker AXSD8 Avance X-ray diffractometer (Germany) with a copper target $(40 \mathrm{kV}, 15 \mathrm{~mA})$. The microstructures of solid residues were recorded using a Cambridge S250MK3 scanning electron microscope (U.K.). The solid residue of the samples degraded to various extents was also prepared using the TGA instrument under nitrogen atmosphere. The materials were heated from room temperature to $600^{\circ} \mathrm{C}$ with a heating rate of $20^{\circ} \mathrm{C} / \mathrm{min}$, and then rapidly cooling the residue to room temperature.

\section{Results and discussion \\ 3.1. Synthesis and characterization of monomer 1 and poly(phosphazene-aryl amides)}

The main objective of this study is to produce novel polyamide with high residual rate (upon $50 \%$ at $600^{\circ} \mathrm{C}$ ), improved solubility and processability without too much sacrificing of their thermal and mechanical properties. The polyamide yields a high char residue at $600^{\circ} \mathrm{C}$ and contains plenty of phosphors element which can be used as surface chemical promoter to achieve good lubricity effect. Cyclic phos- 
phazenes are found with both planar and puckered phosphorus-nitrogen rings. The length of a phosphorus-nitrogen single bond in cyclophosphazene is in the range of 1.47-1.62 $\AA$, and this due to an appreciable contraction. The shortest skeletal bonds are associated with highly electronegative substituents. $\sigma$ and $\sigma-\pi$ bonds are observed in the neutral system. These heterocycles have polar groups, imparting an additional dipole moment to the molecule, and hence they can interact with each other and alter the properties of the polymers containing these groups. So in order to examine the presence of cyclophosphazene groups in the polyamides backbone, aromatic diamine containing phosphazene groups and poly(phosphazenen-aryl amides) were synthesized. Benzene rings were also introduced in the chain in order to render high thermal stability and other properties. The monomer $\mathbf{1}$, which is a new phosphazene aromatic diamine, was synthesized according to Figure 1 through a two-step route by nucleophilic substitution reaction. The nucleophile aromatic substitution for chlorine reaction is a moderate synthesis reaction. On the basis of the results found in the synthesis of the amide model compounds above described, the novel poly(phosphazene-aryl amides) were prepared by solution polycondensation reaction. This synthetic route though it might lead to low molecular weight materials, represents a clean and bland pathway which allows for the control of the chemistry involved.

The chemical structures of the polyphosphazenecontaining oligomer synthesized in the study were characterized by elemental analysis, ${ }^{1} \mathrm{H}$ NMR, ${ }^{31} \mathrm{P}$ NMR and FT-IR spectroscopy. The calculated elemental contents of monomer $\mathbf{1}$ in terms of the formula $\left(\mathrm{C}_{48} \mathrm{H}_{42} \mathrm{~N}_{7} \mathrm{O}_{6} \mathrm{P}_{3}\right)$ were $\mathrm{C}$ 63.65; $\mathrm{H} 4.64$; $\mathrm{N} 10.83$; O 10.61; P 10.27; and the analyzed data show that $\mathrm{C}, \mathrm{H}, \mathrm{N}, \mathrm{O}$ and $\mathrm{P}$ are 63.41, 4.82, 10.69, 10.57 and 10.33 , respectively. It is found that elemental analysis of the synthesized monomer $\mathbf{1}$ is in good agreement with the calculated values. The ${ }^{31} \mathrm{P}$ NMR spectrum of monomer $\mathbf{1}$ recorded at room temperature in DMSO, shows two intense peaks $(\delta=5.64 \mathrm{ppm}$, for the phenol groups linked to the phosphorus atoms; $\delta=7.96 \mathrm{ppm}$, for the phenylamino groups linked to the phosphorus atoms). This experimental evidence denotes that the Chlorine was completely replaced by two groups. Furthermore, in the ${ }^{1} \mathrm{H}$ NMR spectrum of monomer $\mathbf{1}$,

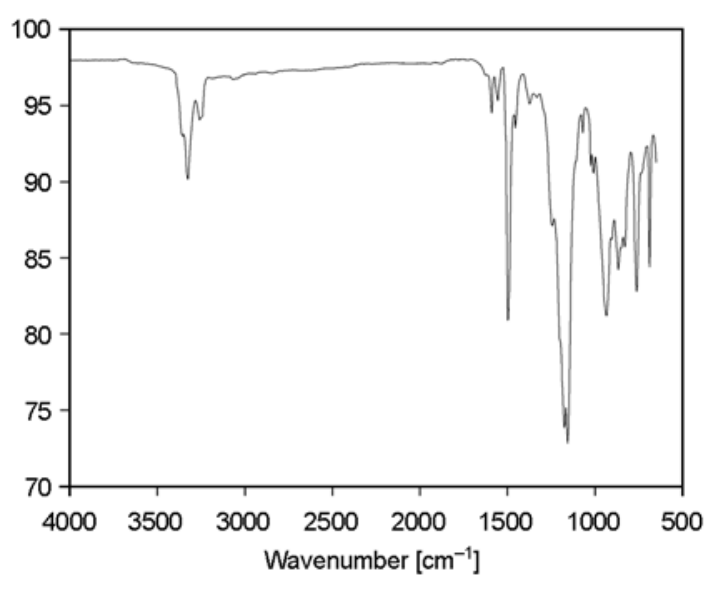

Figure 2. FT-IR spectrum of monomer 1

the chemical shifts corresponding to the phenol ring are found at $\delta=6.89 \mathrm{ppm}$ and $\delta=7.21 \mathrm{ppm} ; \delta=$ $3.04 \mathrm{ppm}$ and $\delta=2.97 \mathrm{ppm}$, as the two doublets prove the presence of the phenylamino groups.

In order to obtain a more complete and reliable chemical characterization, the synthesized monomer 1, was also investigated by FT-IR technique. The spectrum is shown in Figure 2. The monomer shows a strong absorption band at $1230 \mathrm{~cm}^{-1}$ due to the $\mathrm{P}=\mathrm{N}$ stretch, while the absorption band at $875 \mathrm{~cm}^{-1}$ due to the $\mathrm{P}-\mathrm{N}$ stretch. The broad band at 1490 and $1580 \mathrm{~cm}^{-1}$ corresponds to the benzene ring in AOPP. It also shows strong absorption peaks at $1050 \mathrm{~cm}^{-1}$, at 940 and $3320 \mathrm{~cm}^{-1}$ due to the $\mathrm{C}-\mathrm{O}-\mathrm{C}$ stretch, $\mathrm{P}-\mathrm{O}-\mathrm{Ar}$ stretch and $\mathrm{N}-\mathrm{H}$ stretching, respectively.

The FT-IR spectra of the poly(phosphazene-aryl amides) shown in Figure 3 present the characteristic absorption bands due to eh stretching of the - NHamide groups along the chain in the range 3000$3500 \mathrm{~cm}^{-1}$, while the stretching of the polyphosp-

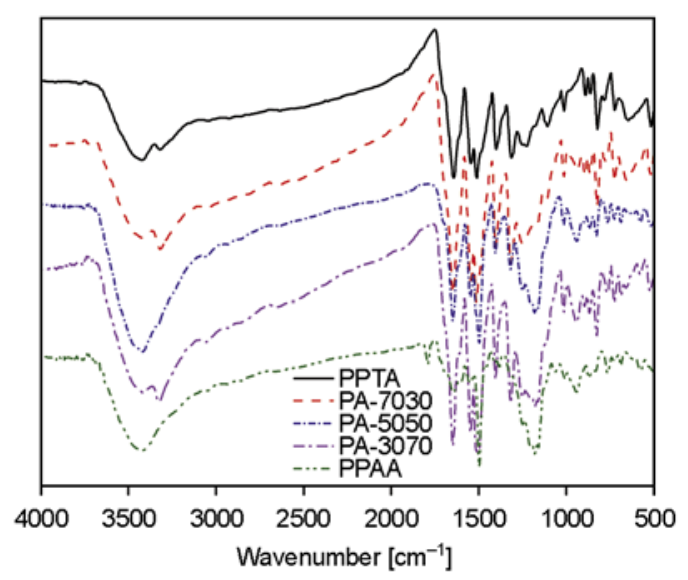

Figure 3. FT-IR spectrum of the poly(phosphazene-aryl amides) 
hazene groups give a wide band at $1200 \mathrm{~cm}^{-1}$. Characteristic bands due to the aromatic polyamides were observed, for example, at $1655 \mathrm{~cm}^{-1}$ [amide I $\left(\mathrm{C}=\mathrm{O}\right.$ stretching mode)] and $1530 \mathrm{~cm}^{-1}$ [amide II (coupling of $\mathrm{C}-\mathrm{N}$ stretching and $\mathrm{N}-\mathrm{H}$ in-plane bending modes)]. Very similar FT-IR spectra were achieved for fully aromatic polyphosphazene polyamides (Table 1).

In conclusions, the inspection reveals that the novel poly(phosphazene-aryl amides) synthesized and discussed in the present work can be well characterized and distinguished applying these powerful methods.

\subsection{Solubility and Intrinsic viscosity of the poly(phosphazene-aryl amide)}

The solubility of the fully aromatic poly(phosphazene-aryl amide) was tested in various solvents at $1 \mathrm{wt} \%$ concentration and the results are summarized in Table 2.

The new polyamide (PPAA) was readily soluble in highly polar solvents such as DMF, DMAc, DMSO and NMP at room temperature or upon heating. The high-solubility nature of PPAA can be explained by the presence of polypolyphosphazene groups. Because of these voluminous groups, the packing of the polymer chains in tight structures through hydrogen bonding between amide groups is prevented and, consequently, the solvent molecules can easily diffuse into the polymer chain. The increased solubility of these polyamides is mainly due to their amorphous character. And the solubility of the materials is similar to those of conventional maramids. All the X-ray diffraction diagrams of polyamides gave predominately amorphous patterns (Figure 4). The stereo structure of PPAA is shown in Figure 4.

In order to estimate the molecular weights of the polyamide products, their intrinsic viscosities $\left(\eta_{\text {inh }}\right)$ were measured using an Ubbelohde viscometer

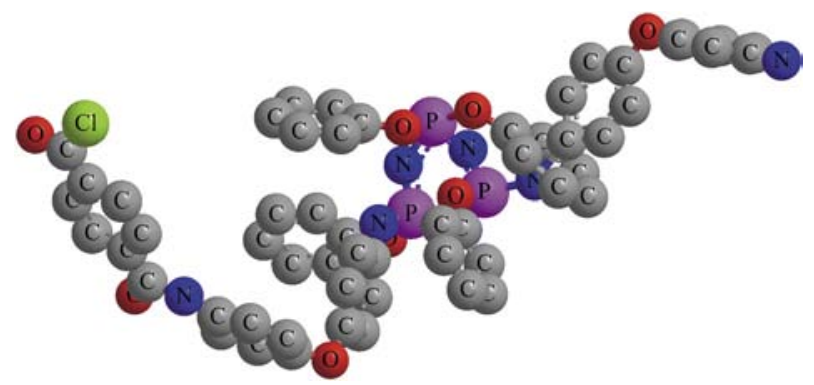

Figure 4. Model of a macromolecule fragment incorporating 1 repeating units of PPAA

Table 3. Inherent viscosity $\left(\eta_{\text {inh }}\right)$ and GPC valuble of polyamides

\begin{tabular}{|c|c|c|c|}
\hline Polymer code & $\eta_{\text {inh }}{ }^{1} \cdot\left[\mathrm{dl} \cdot \mathrm{g}^{-1}\right]$ & $\mathrm{M}_{\mathrm{w}}{ }^{2} \cdot\left[\mathrm{g} \cdot \mathrm{mol}^{-1}\right]$ & PD \\
\hline PPTA & 3.16 & - & - \\
\hline PA-7030 & 2.31 & - & - \\
\hline PA-5050 & 1.62 & - & - \\
\hline PA -3070 & 0.98 & - & - \\
\hline PPAA & 0.75 & 38300 & 1.78 \\
\hline
\end{tabular}

${ }^{1}$ Inherent viscosity measured at a concentration of $0.5 \mathrm{~g} / \mathrm{dl}$ in concentrated sulfuric acid, at $30 \pm 0.2^{\circ} \mathrm{C}$.

${ }^{2}$ Measured by GPC in DMF; polystyrene was used as standard.

with concentrated sulfuric acid at $30 \pm 0.2^{\circ} \mathrm{C}$. Meanwhile, the weight-average molecular weights $\left(M_{\mathrm{w}}\right)$ and polydispersities $\mathrm{PD}\left(=M_{\mathrm{w}} / M_{\mathrm{n}}\right)$ of the polyamide was estimated by GPC measurements in DMF. The values are all listed in Table 3. The testing data indicate that the polymers have quite a narrow molecular weight distribution and a low quantity of oligomers.

\subsection{Crystallinity of our synthesized poly(phosphazene-aryl amides)}

In order to study the crystalline or amorphous nature of the polyamides, WAXD measurements at room temperature were performed. Typical wide-angle $\mathrm{X}$-ray diffractograms of the powder polyamides are illustrated in Figure 5. The powder samples were pulps which obtained after precipitation from solution (synthesis), without through spinning and fiber processing processes. From the curves we can see

Table 2. Solubility behaviour ${ }^{1}$ of the polyamides in various solvents ${ }^{2}$

\begin{tabular}{|l|c|c|c|c|c|c|c|}
\hline Polymer code & $\mathbf{C H C l}_{\mathbf{3}}$ & THF & DMF & DMAc & NMP & DMSO & $\mathbf{H}_{2} \mathbf{S O}_{4}$ \\
\hline PPTA & - & - & - & - & - & - & + \\
\hline PA-7030 & - & - & - & - & - & - & + \\
\hline PA-5050 & - & - & - & - & - & \pm & + \\
\hline PA -3070 & - & - & \pm & \pm & \pm & \pm & + \\
\hline PPAA & - & - & \pm & \pm & + & + & + \\
\hline
\end{tabular}

${ }^{1}$ Solubility: $(+)$ soluble at room temperature; $( \pm)$ soluble with warming or swollen; $(-)$ insoluble.

${ }^{2}$ THF: tetrahydrofuran; DMF: $N, N$-dimethyl formaide; DMAc: $N, N$-dimethyl acetamide; NMP: $N$-methyl pyrrolidone; DMSO: dimethyl sulfoxide. 


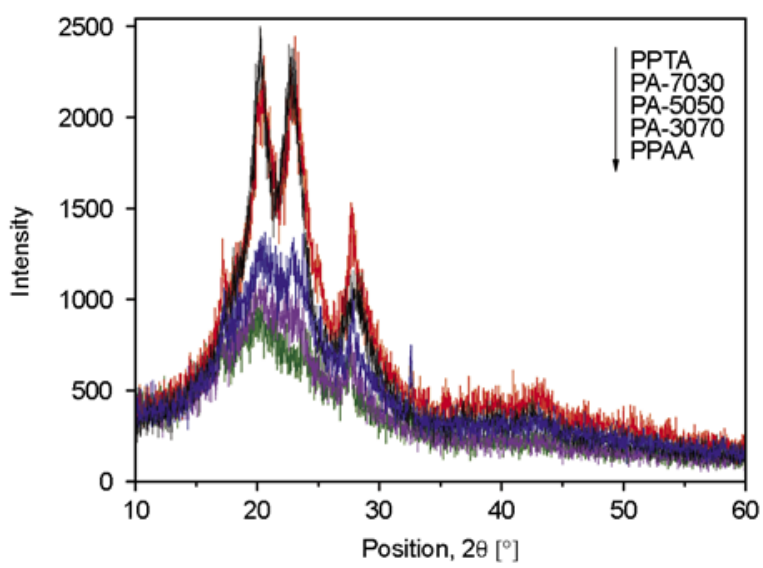

Figure 5. XRD patterns of the polyamides

that there are two dispersion peaks at $2 \theta=20.5$ and $23.5^{\circ}$ for PPTA, which indicates PPTA has low crystallinity. But, there are no sharp peaks in the PPAA. It proves that PPAA is amorphous. This can be attributable to the introduction of polyphosphazene groups, which weakens the regular structure of molecular chains, reduces the rigidity and therefore disrupts the crystallization capacity of the polyamides. Thus, the amorphous structure of this polyamide also reflected in its excellent solubility as can be observed in Table 2 .

\subsection{Thermal properties of the polyamides}

The thermal properties and stability of all poly (phosphazene-aryl amides) were studied by DSC and TGA techniques, respectively, and the data are reported in Table 4. As can be observed, in Table 2 the poly(phosphazene-aryl amides) are amorphous samples with a glass transition $\left(T_{\mathrm{g}}\right)$ of $85^{\circ} \mathrm{C}$. For the compounds PA-7030, PA-5050 and PA-3070 we expect a high $T \mathrm{~g}$ values similar to PPTA, which have a $T_{\mathrm{g}}$ values higher than $120^{\circ} \mathrm{C}$. And the $T_{\mathrm{g}}$ values decreased with decreasing rigidity and symme-

Table 4. Data results for the polyamides

\begin{tabular}{|l|c|c|c|c|}
\hline \multicolumn{1}{|c|}{ Code } & $\begin{array}{c}\mathbf{T}_{\mathbf{0}} \\
{\left[{ }^{\circ} \mathbf{C}\right]^{\mathbf{1}}}\end{array}$ & $\begin{array}{c}\mathbf{T}_{\mathbf{1 0}} \\
{\left[{ }^{\circ} \mathbf{C}\right]^{\mathbf{2}}}\end{array}$ & $\begin{array}{c}\mathbf{C h a r} \text { yield } \\
{\left[\mathbf{\%}^{\mathbf{3}}{ }^{\mathbf{3}}\right.}\end{array}$ & $\begin{array}{c}\mathbf{T}_{\mathbf{g}} \\
{\left[{ }^{\circ} \mathbf{C}^{\mathbf{4}}\right.}\end{array}$ \\
\hline PPTA & 455.3 & 482.8 & 58.68 & 341 \\
\hline PA-7030 & 237.8 & 308.6 & 66.55 & 195 \\
\hline PA-5050 & 198.6 & 298.7 & 61.27 & 157 \\
\hline PA-3070 & 193.1 & 291.0 & 56.56 & 146 \\
\hline PPAA & 180.7 & 263.4 & 55.58 & 85 \\
\hline
\end{tabular}

${ }^{1}$ Initial decomposition temperature recorded by TGA at a heating rate of $20^{\circ} \mathrm{C} / \mathrm{min}$ in $\mathrm{N}_{2}$.

${ }^{2}$ Temperature at which $10 \%$ weight loss is observed.

${ }^{3}$ Anaerobic residual weight at $600^{\circ} \mathrm{C}$.

${ }^{4}$ Glass transition temperature measured on DSC at a heating rate of $10^{\circ} \mathrm{C} / \mathrm{min}$ in $\mathrm{N}_{2}$.

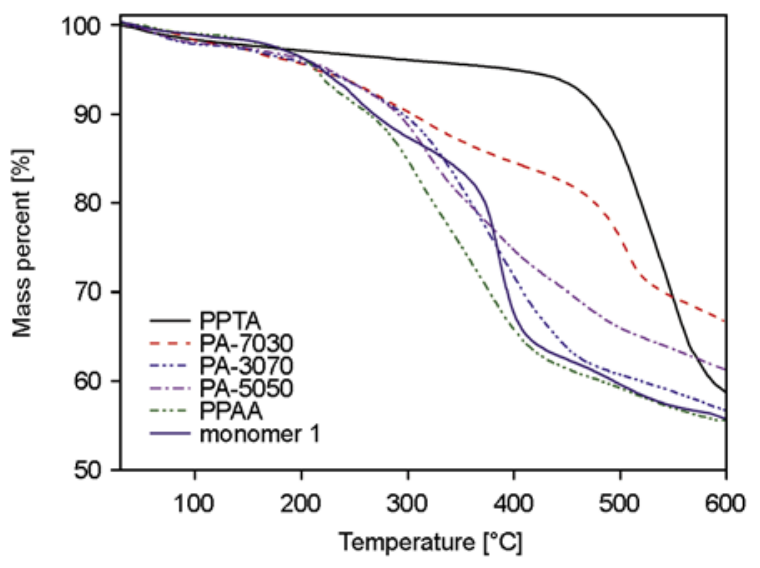

Figure 6. TGA curves for the polyamides

try of the polymer backbone, which is due to the increase of monomer $\mathbf{1}$ content.

The thermal stabilities of the poly(phosphazenearyl amides) were evaluated by TGA at $20^{\circ} \mathrm{C} / \mathrm{min}$ up to $600^{\circ} \mathrm{C}$ under nitrogen. Figure 6 shows a typical temperature dependence profile of the polyamides weight loss. The PPTA sample presents a maximum rate of the thermal decomposition temperature at $512^{\circ} \mathrm{C}$, yielding a char residue of $58.68 \%$ at $600^{\circ} \mathrm{C}$, while a char residue of $55.58 \%$ at $600^{\circ} \mathrm{C}$ was observed in the thermal decomposition of the PPAA, of $66.55 \%$ for the PA-7030, of $61.27 \%$ for the PA-5050, and of $56.56 \%$ for the PA-3070. The two decomposition steps for PPAA occur at $180.7^{\circ} \mathrm{C}$ and $271.5^{\circ} \mathrm{C}$, respectively. The weight loss of PPTA at around $100-150^{\circ} \mathrm{C}$ is due to the evaporation of absorbed moisture. The PPAA loss $7 \%$ of the sample in the temperature range $150-250^{\circ} \mathrm{C}$, and another $35.42 \%$ in the range $250-600^{\circ} \mathrm{C}$. PPTA sample loss $35.32 \%$ of the sample in temperature range $455-600^{\circ} \mathrm{C}$. Comparing the curves in Figure 5, it emerges that the first decomposition step of the monomer 1 occurs around $150-350^{\circ} \mathrm{C}$. This is attributed to the less stable of the $\mathrm{P}-\mathrm{O}-\mathrm{C}$ bone linkage, as reported for other polymeric systems containing similar polyphosphazene groups [19, 20]. It also determines the lower thermal stability of the fully aromatic polyphosphazene polyamides with respect to their no-polyphosphazene samples.

A pleasant surprise from the curves, we can confirm that PA-3070 and PA-5050 show higher char yields than PPTA. It can be readily attributed to the inherent thermal-stability properties of the polyphosphazene cores. With the presence of a small amount of polyphosphazene diamine, cross-linking and gela- 
tion occurs in the decomposition. As the network structure and gelation can inhibit the production of small molecules, the higher char yield is an effect of achieving synergy between the individual units.

\subsection{Thermal degradation properties}

The TGA results indicated that polyphosphazenes play an important roals in increasing the themal stability of poly(phosphazene-aryl amides) at elevated temperatures. However, its stability at lower temperatures decreases. To further investigate their influence, the residues of the polyamides samples obtained from the TGA measurements, from room temperature to $600^{\circ} \mathrm{C}$ with a heating rate of $20^{\circ} \mathrm{C} / \mathrm{min}$ in air, were analyzed by FT-IR and SEM. Figure 7 illustrates the FT-IR spectra of the pyrolysis residues. In the FT-IR spectra of poly(phosphazene-aryl amides) samples, the absorption peak at $940 \mathrm{~cm}^{-1}$ due to $\mathrm{P}-\mathrm{O}-\mathrm{C}$ bone and the absorption peak at $1500 \mathrm{~cm}^{-1}$ due to $\mathrm{N}-\mathrm{H}$ bending and $\mathrm{C}-\mathrm{N}$ stretching disappear. The characteristic absorption peaks for $\mathrm{P}=\mathrm{N}$ at about $1230 \mathrm{~cm}^{-1}, \mathrm{P}-\mathrm{N}$ at $875 \mathrm{~cm}^{-1}$ and for ether group at 1180 also disappear. However, a few new peaks appear at $1056 \mathrm{~cm}^{-1}$, which might be ascribed to the generation of $\mathrm{P}-\mathrm{O}-\mathrm{P}$. Similar to the mechanism which has been reported [20,21]. The obtained structure could act as an acid catalyst, accelerating the cleavage of side groups and the breaking of ether groups in poly(phosphazene-aryl amides). Then, the polyamides react to form more stable structures. The appearance of $\mathrm{P}-\mathrm{O}-\mathrm{P}$ group is considered as crosslinking to different species, resulting in the formation of complex phosphorus structures. This is why the thermal degradation of the poly(phosphazene-aryl amides) is much slower than PPTA at above $500^{\circ} \mathrm{C}$. The morphology of the solid residues was observed by Scanning Electron Microscope (Figure 8). The surface of monomer 1 (Figure $8 \mathrm{f}$ ) residues exhibits a porous texture reverse to the lump of PPTA (Figure 8a). Comparing with the pictures (Figure 8), we can see that the granular of the solid residues gradual disappearance with the increase of monomer 1 content. The surface layer of PPAA solid residues has been grumous, for the syneresis of $\mathrm{P}-\mathrm{O}-\mathrm{P}$ took place. It proves that the cyclotriphosphazene moieties produce phosphoric acid or metaphosphoric acid during pyrolysis which acts in the condensed phase promoting char formation on the surface.

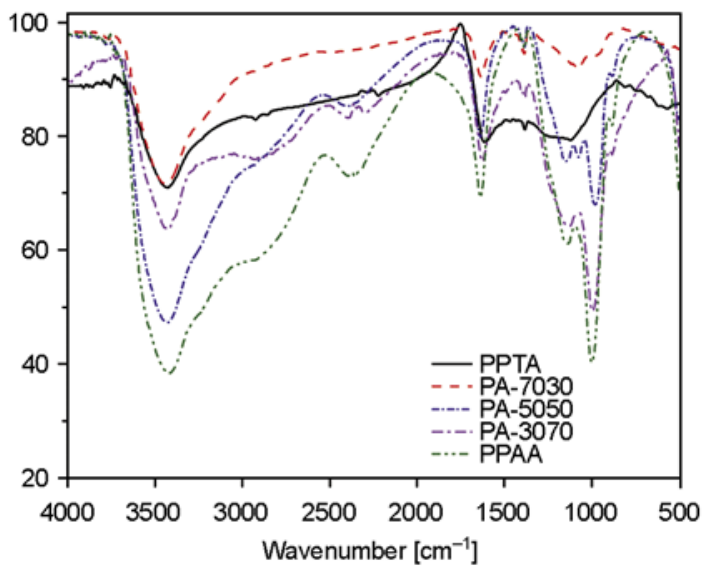

Figure 7. FT-IR curves for the solid residues of poly(phosphazene-aryl amides) after pyrolysis

\subsection{LOI values of poly(phosphazene-aryl amides)}

Char yield can be applied as decisive factor for estimating limiting oxygen index (LOI) of the polymers based on Van Krevelen and Hoftyzer equation [22] (Equation (1)):

$\mathrm{LOI}=17.5+0.4 \mathrm{CR}$

where CR - Char yield (the data are reported in Table 2). Thus, the LOI values are speculated from TGA date, not experimental burning data.

The values of LOI are plotted in Figure 9. The average values of LOI for the two polyamides are represented by the dotted lines connecting the points for the two pure components (PPTA and PPAA). The poly(phosphazene-aryl amides) had LOI values calculated derived from their char yield was high than 39. On the basis of LOI values, such macromolecules can be classified as self-extinguishing polymers.

\section{Conclusions}

New kinds of poly(phosphazene-aryl amides) were prepared by polycondensation in solution of the our synthesized monomer 1 with terephthaloyl dichloride. The monomer $\mathbf{1}$ was characterized by elemental analysis, ${ }^{1} \mathrm{H}$ NMR, ${ }^{31} \mathrm{P}$ NMR and FT-IR techniques to verify its chemical structure. The results confirm this successful synthesis. The investigation on thermal properties shows that the thermal stability of our synthesized fully aromatic polyphosphazene polyamides are strong determined by the presence of the polyphosphazene groups in the 


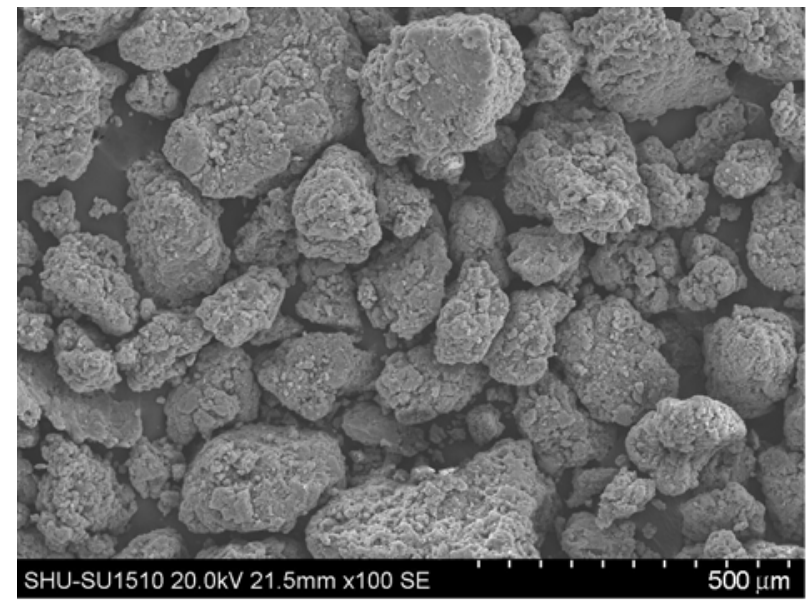

a)

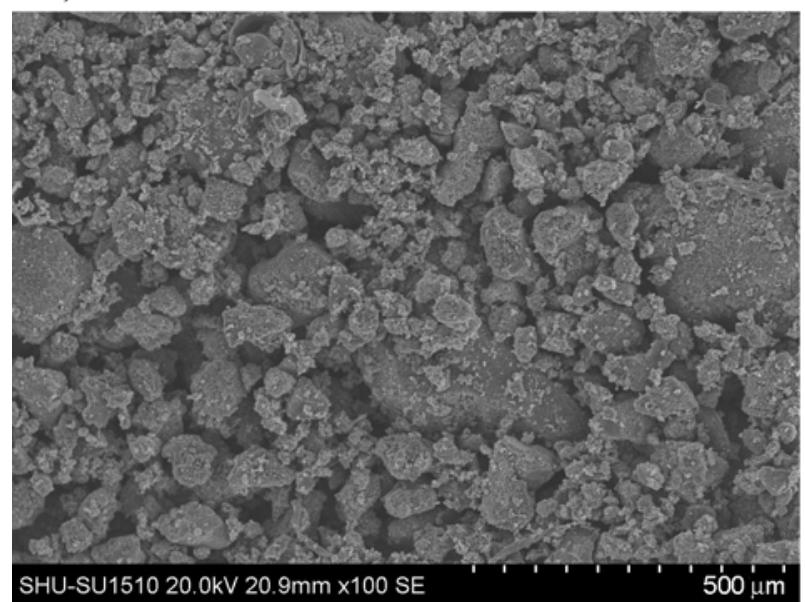

c)

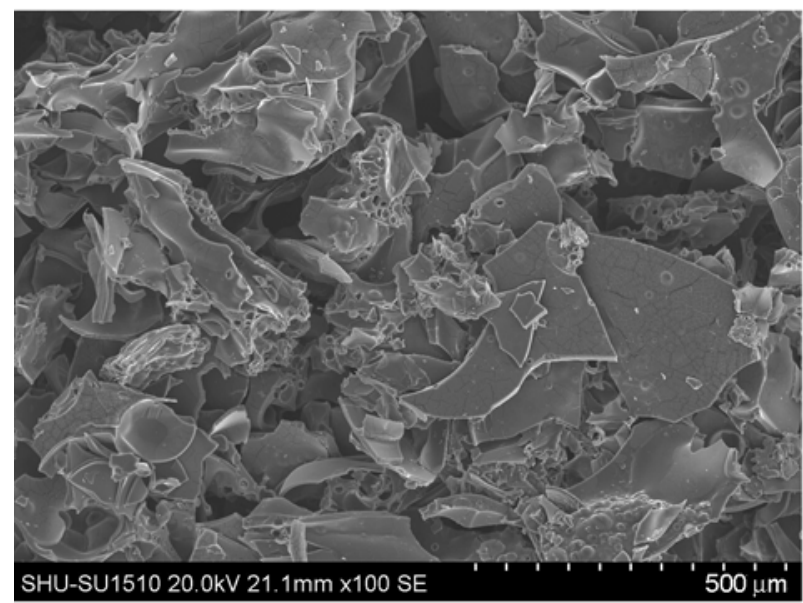

e)

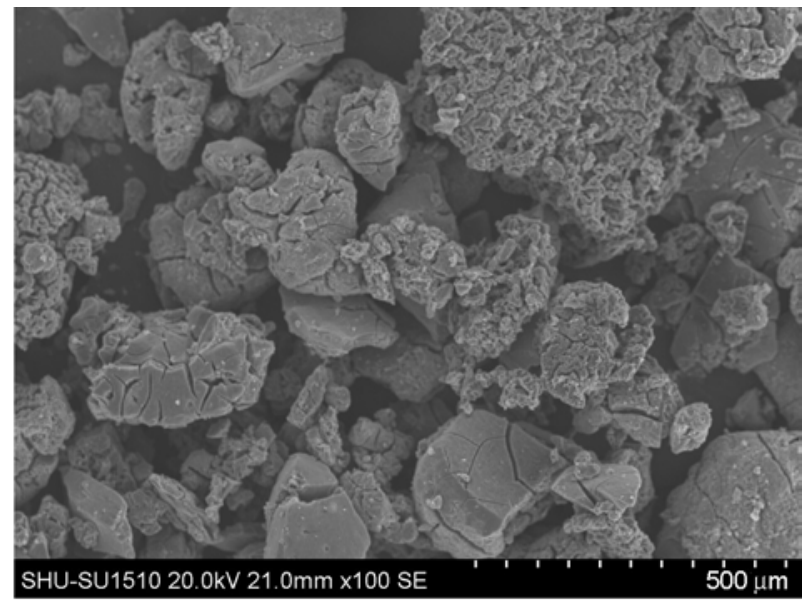

b)

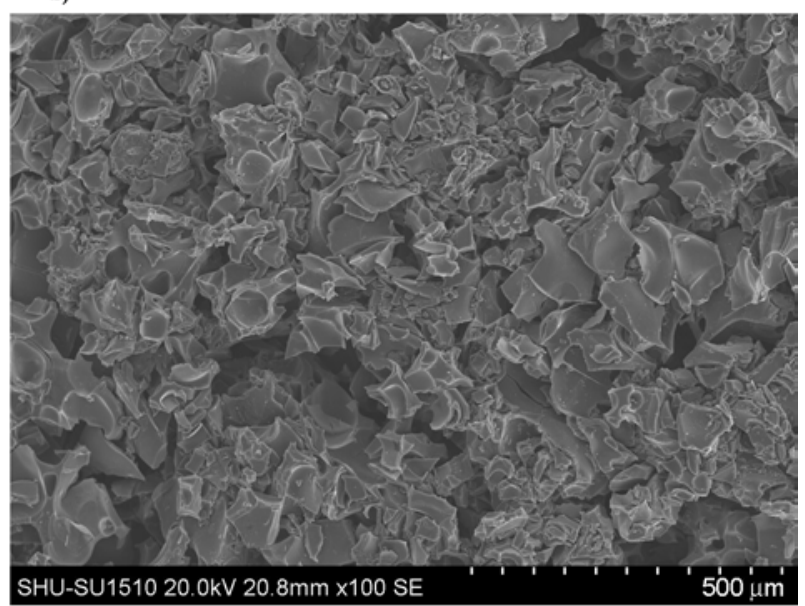

d)

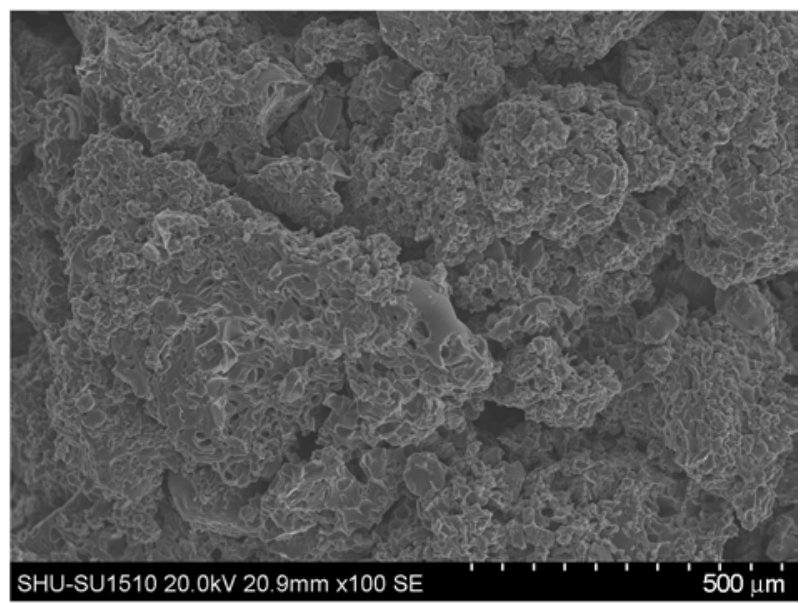

f)

Figure 8. Morphology of the solid residues of the composites after pyrolysis: (a) PPTA, (b) PA-7030, (c) PA-5050, (d) PA-3070, (e) PPAA, (f) monomer 1

polymer chains. They thermally degradation in two steps, and this behavior may be due to the decomposition of the $\mathrm{P}-\mathrm{O}-\mathrm{C}$ bone and aromatic bones. The surface of monomer $\mathbf{1}$ solid residues exhibits a porous texture with the scanning electron micro- scope. During pyrolysis, polyphosphazene not only acted in the condensed phase to promote char formation at the surface but also promoted gelation to restrain pyrolysis indirectly. 


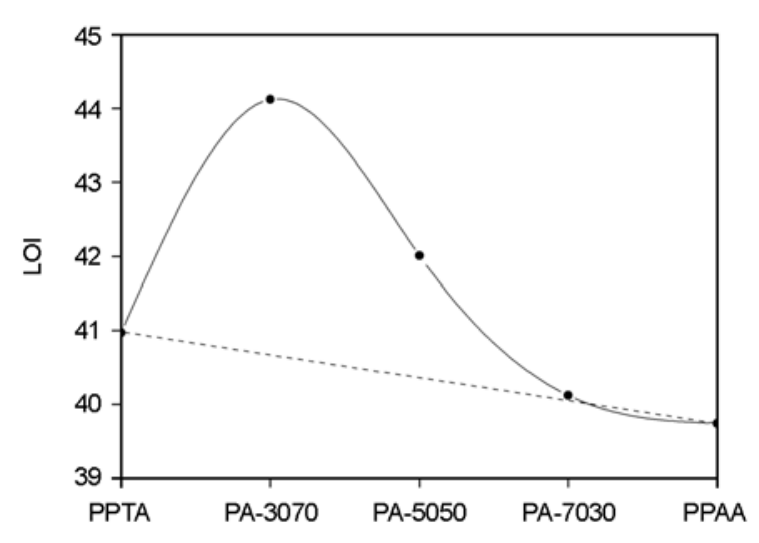

Figure 9. LOI values of poly(phosphazene-aryl amides)

\section{Acknowledgements}

We are thankful to the National Natural Science Foundation of China (Fund No. 50975167) and Shanghai Leading Academic Discipline Project (Project Number: S30107) for the continuing support to this research.

\section{References}

[1] García J. M., García F. C., Serna F., de la Peña J. L.: High-performance aromatic polyamides. Progress in Polymer Science, 35, 623-686 (2010). DOI: 10.1016/j.progpolymsci.2009.09.002

[2] Quadrini F., Squeo E. A., Tagliaferri V.: Machining of glass fiber reinforced polyamide. Express Polymer Letters, 1, 810-816 (2007).

DOI: 10.3144/expresspolymlett.2007.112

[3] Yagci H., Mathias L. J.: Synthesis and characterization of aromatic polyamides and polyimides from trimethyland di-t-butylhydroquinone-based ether-linked diamines. Polymer, 39, 3779-3786 (1998).

DOI: $10.1016 / \mathrm{S} 0032-3861(97) 10378-0$

[4] Meléndez A., de la Campa J. G., de Abajo J.: Polyisophthalamides with phenoxy pendant groups. Polymer, 29, 1142-1145 (1988).

DOI: 10.1016/0032-3861(88)90030-4

[5] Sava I., Iosip M-D., Bruma M., Hamciuc C., Robison J., Okrasa L., Pakula T.: Aromatic polyamides with pendent acetoxybenzamide groups and thin films made therefrom. European Polymer Journal, 39, 725-738 (2003).

DOI: 10.1016/S0014-3057(02)00295-1

[6] Tamami B., Yeganeh H.: Synthesis and properties of novel aromatic polyamides based on 4-aryl-2,6-bis(4chlorocarbonylphenyl) pyridines. European Polymer Journal, 38, 933-940 (2002).

DOI: 10.1016/S0014-3057(01)00264-6

[7] Wang W-Z., Zhang Y-H.: Environment-friendly synthesis of long chain semiaromatic polyamides. Express Polymer Letters, 3, 470-476 (2009).

DOI: $\underline{10.3144 / \text { expresspolymlett.2009.58 }}$
[8] Rao Y., Waddon A. J., Farris R. J.: Structure-property relation in $\operatorname{poly}(p$-phenylene terephthalamide) (PPTA) fibers. Polymer, 42, 5937-5940 (2001).

DOI: $10.1016 / \mathrm{S} 0032-3861(00) 00905-8$

[9] Yagci H., Mathias L. J.: Synthesis and characterization of aromatic polyamides and polyimides from trimethyland di-t-butylhydroquinone-based ether-linked diamines. Polymer, 39, 3779-3786 (1998). DOI: $10.1016 / \mathrm{S} 0032-3861(97) 10378-0$

[10] Klaus E. E., Li H., Chen C. I., Duda J. L.: Wear as measured with a four-ball wear tester using fully flooded and vapor phase with condensation lubrication methods. Wear, 162, 944-949 (1993).

DOI: 10.1016/0043-1648(93)90099-8

[11] Argibay N., Keith J. H., Krick B. A., Hahn D. W., Bourne G. R., Sawyer W. G.: High-temperature vapor phase lubrication using carbonaceous gases. Tribology Letters, 40, 3-9 (2010). DOI: $10.1007 / \mathrm{s} 11249-009-9514-7$

[12] Sung D., Gellman A. J.: The surface chemistry of alkyl and arylphosphate vapor phase lubricants on Fe foil. Tribology International, 35, 579-590 (2002). DOI: $10.1016 / \mathrm{S} 0301-679 \mathrm{X}(02) 00045-2$

[13] Abdelmaksoud M., Bender J. W., Krim J.: Nanotribology of a vapor-phase lubricant: A quartz crystal microbalance study of tricresylphosphate (TCP) uptake on iron and chromium. Tribology Letters, 13, 179-186 (2002).

[14] Jin Y. S., Zhou C. H.: The effectiveness of high temperature lubrication by in situ formation of graphite/ $\mathrm{MoS}_{2}$ films. Wear, 205, 77-87 (1997).

DOI: 10.1016/S0043-1648(96)07308-5

[15] Stirniman M. J., Falcone S. J.: The ideality of polydisperse perfluoropolyether lubricants with application to physical vapor deposition. Tribology Letters, 8, 171177 (2000).

DOI: 10.1023/A:1019135218167

[16] Smith C. J., Furey M. J., Kajdas C.: An exploratory study of vapor-phase lubrication of ceramics by monomers. Wear, 181-182, 581-593 (1995). DOI: 10.1016/0043-1648(95)90174-4

[17] Liu W., Klaus E. E., Duda J. L.: Wear behaviour of steel-on- $\mathrm{Si}_{3} \mathrm{~N}_{4}$ and $\mathrm{Si}_{3} \mathrm{~N}_{4}$-on- $\mathrm{Si}_{3} \mathrm{~N}_{4}$ systems with vapor phase lubrication of oleic acid and TCP. Wear, 214, 207-211 (1998).

DOI: 10.1016/S0043-1648(97)00253-6

[18] Brandt K., Jekel A. P., Meetama A., Van De Grampel J. C.: Syntheses and structures of precursors in the polycondensation of hexachlorocyclotriphosphazene and hydroquinone. Inorganica Chimica Acta, 157, 251258 (1989). DOI: $10.1016 / \mathrm{S} 0020-1693(00) 80549-6$

[19] EI Gouri M., EI Bachiri A., Hegazi S. E., Rafik M., El Harfi A.: Thermal degradation of a reactive flame retardant based on cyclotriphosphazene and its blend with DGEBA epoxy resin. Polymer Degradation and Stability, 94, 2101-2106 (2009).

DOI: $10.1016 /$ j.polymdegradstab.2009.08.009 
[20] Maynard S. J., Sharp T. R., Haw J. F.: Thermal degradation chemistry of poly(diphenoxyphosphazene). Macromolecules, 24, 2794-2799 (1991).

DOI: $10.1021 / \mathrm{ma} 00010 \mathrm{a} 024$
[21] Liu R., Wang X.: Synthesis, characterization, thermal properties and flame retardancy of a novel nonflammable phosphazene-based epoxy resin. Polymer Degradation and Stability, 94, 617-624 (2009).

DOI: 10.1016/j.polymdegradstab.2009.01.008

[22] Van Krevelen D. W., Hoftyzer R. J.: Properties of polymer. Elsevier, New York (1976). 